\title{
EMP stars with high mass IMF and hierarchical galaxy formation
}

\author{
Yutaka Komiya ${ }^{1}$, Takuma Suda ${ }^{2}$, Asao Habe ${ }^{3}$, \\ and Masayuki Y. Fujimoto ${ }^{3}$
}

\author{
${ }^{1}$ National Astronomical Observatory of Japan, 2-21-1 Osawa, Mitaka, Tokyo, 181-8588, Japan \\ ${ }^{2}$ Astrophysics Group, EPSAM, Keele University, Keele, Staffordshire ST5 5BG, UK \\ ${ }^{3}$ Department of Cosmoscience, Hokkaido University, Sapporo, Hokkaido 060-0810, Japan
}

\begin{abstract}
Extremely metal-poor (EMP) stars in the Galactic halo are stars formed in the very early stage of the chemical evolution of the Galaxy. In previous study, we proposed that typical mass of EMP stars are massive, based on observations of carbon-enhanced EMP stars. In this study, we build a merger tree of the Galaxy semi-analytically and follow the chemical evolution along the merger tree. We also consider the effect of binary and high-mass initial mass function(IMF). Resultant theoretical metallicity distribution function (MDF) and abundance distribution are compared with observed metal-poor halo stars.
\end{abstract}

Keywords. stars: abundances, stars: Population II, Galaxy: evolution, Galaxy: halo

\section{Introduction}

In the Galactic halo, many EMP stars with metallicity $[\mathrm{Fe} / \mathrm{H}]<-2.5$ are detected thanks to large scaled surveys Beers et al. (1992), Christlieb et al. (2001). These stars are expected to be probes to the first stars and galaxy formation in the early universe.

\section{IMF of EMP stars}

In previous studies (Komiya et al. 2007, Komiya et al. 2009a), we gives constraints on IMF of EMP stars from statistics of carbon rich stars $([\mathrm{C} / \mathrm{Fe}]>\sim 0.5)$ (CEMP stars). Observed CEMP stars shows following peculiar features. Fraction of CEMP stars among EMP stars is $(\sim 20-25 \%$ Rossi et al. 1999). It is much larger than the fraction of carbon rich stars among Population I and II stars. There are two groups of CEMP stars, with and without enhancement of s-process elements referred to as CEMP-s and CEMP-nos, respectively (Aoki et al. 2002). Observationally, number ratio between CEMP-nos stars to CEMP-s stars is $\sim 1 / 3-1$.

CEMP stars thought to be formed through binary mass transfer from AGB primaries. Theoretical studies about evolution of EMP stars shows that hydrogen mixing event for $[\mathrm{Fe} / \mathrm{H}]<-2.5$ and carbon (and s-elements) enrichment process (Fujimoto et al. 1990, Suda et al. 2004). These studies shows that CEMP-s stars are produced from $0.8-3 M_{\odot}$ primary and CEMP-nos stars are produced from $4-6 M_{\odot}$ primary.

From obervations, we can estimate number fraction of binary with $0.8-3 M_{\odot}$ primary and 4-6 $M_{\odot}$ primary. Large fraction of CEMP stars, especially CEMP-nos stars, indicate large fraction of intermdiate massive stars. We assume lognormal IMF and flat mass-ratio distribution for binary, and give constraints on medium mass, $M_{\mathrm{md}}$, and dispersion, $\sigma$, of the IMF. As a result, only high mass IMFs with $M_{\mathrm{md}}>7$ are consistent with observation.

Another constraint is from averaged iron yield of EMP stars. To pollute the Galaxy to $[\mathrm{Fe} / \mathrm{H}]=-2.5,5 \times 10^{5} M_{\odot}$ of iron is required. On the other hand, number of massive 
EMP stars in the early universe can be estimated from number of EMP stars observed by large scale surveys when we assume the IMF. Thus, from observation, we can estimate the averaged iron yield, $\left\langle Y_{F e}\right\rangle$, per massive EMP stars as a function of $M_{\mathrm{md}}$.

Onthe other hand, it is said that iron yield of type II supernova is $\sim 0.07 M_{\odot}$ from observations. Theoretical studies about supernova nucleosynthesis derive the dependence of the iron yield on the mass of progenitor stars (Woosley \& Weaver 1995, Umeda \& Nomoto 2002). We calculate IMF weighted average of iron yield as a function of $M_{\mathrm{md}}$.

These two estimation of averaged iron yield $\left\langle Y_{F e}\right\rangle$ should be same. As a result, $\left.<Y_{F e}\right\rangle$ from observation of EMP stars are consistent with theoretical value at $M_{\mathrm{md}} \sim$ $10 M_{\odot}$. From two constraints, from CEMP stars and iron yield, we conclude that $M_{\mathrm{md}} \sim$ $10 M_{\odot}$ and $\sigma \sim 0.4$ for EMP stars.

\section{Hierarchical chemical evolution}

High mas IMF thought to be affect chemical evolution. We calculate chemical evolution with high-mass IMF. In the $\Lambda$ CDM cosmology, galaxy is formed hierarchically. We build merger tree by the method of Somerville \& Kolatt (1999), and calculate star formation history and chemical evolution of mini-halos along the tree (Komiya et al. 2009b). We register all the individual Population III and EMP stars and follow their evolution. We assume instantaneous mixing inside each halos and constant star formation efficiency.

Our model well reproduce the metallicity distribution function of EMP stars. Little stars are distributed at $-\infty<[\mathrm{Fe} / \mathrm{H}]<-4$ because metal abundance of mini-halos becomes $[\mathrm{Fe} / \mathrm{H}]>-4$ after first Type II SNe in their host mini-halo. It is consistent with the observational scarcity of stars with $[\mathrm{Fe} / \mathrm{H}]<-4$. Absolute number of EMP stars as well as form of MDF consistent with observations, for the high-mass IMF.

For the abundance of relative element abundances, dependence of IMF is relatively small and dependence on the assumptions about supernova yield is larger. For example, resultant distribution of alpha element abundance, $[\alpha / \mathrm{Fe}]$, with high-mass IMF are similar to Salpeter IMF. Ont the other hand, when we assume that stars with $6-8 M_{\odot}$ become carbon defragration supernova (Nomoto et al. 1984), they eject large amoujnt of iron and decrease the $[\alpha / \mathrm{Fe}]$. Hypernova also yield larger amount of iron than normal supernova and decrease $[\alpha / \mathrm{Fe}]$ (Kobayashi et al. 2006).

We conclude that observation total number and MDF of EMP stars is imprtant to estimate IMF of low-metallicity stars.

\section{References}

Aoki, W., Norris, J. E., Ryan, S. G., Beers, T. C., \& Ando, H., 2002, ApJ, 567, 1166

Beers, T. C., Preston, G. W., \& Shectman, S. A. 1992, aj, 103, 1987

Christlieb, N., Green, P. J., et al. 2001, A\& A, 375, 366

Fujimoto, M. Y., Iben, I. Jr., \& Hollowell, D. 1990, ApJ, 349, 580

Kobayashi, C., Umeda, H., Nomoto, K., Tominaga, N., \& Ohkubo, T. 2006, 653, 1145

Komiya, Y., Suda, T., Minaguchi, H., et al. 2007 ApJ, 658, 367

Komiya, Y., Suda, T., \& Fujimoto, Y. M. 2009a, ApJ, 694, 1577

Komiya, Y., Suda, T., \& Fujimoto, Y. M. 2009b, ApJL, 696, L79

Nomoto, K., Thielemann, F.-K., \& Yokoi, K., 1984, 286, 644

Rossi, S. C. F., Beers, T. C., \& Sneden, C. 1999, ASP Conf. Ser. 165, 65, 264

Somerville, R. S. \& Kolatt, T. S. 1999, MNRAS, 305, 1

Suda, T., Aikawa, M., Machida, et al. 2004, ApJ, 611, 476

Umeda, H. \& Nomoto, K. 2002, ApJ, 565, 385

Woosley, S. E. \& Weaver, T. A. ApJS, 101, 181 